\title{
UNE NOUVELLE ESPÈCE DE NÉMATODE CAPILLARIINAE PARASITE DE SORICIDÉS (MAMMALIA, INSECTIVORA) AU NÉPAL
}

\author{
J.-L. JUSTINE
}

RÉSUMÉ

Capillaria ohbayashii n. sp. est décrit à partir de spécimens parasites de l'appareil urinaire d'Insectivores Soricidés récoltés au Népal chez Soriculus nigrescens (hôte-type) et chez Soriculus caudatus, Suncus murinus et Chimarrogalle himalayaca. Le mâle (longueur moyenne du corps $8933 \mu \mathrm{m}$ ) est caractérisé par l'absence d'ailes latéro-caudales, un cirre inerme, une extrémité caudale recourbée vers la face ventrale, une bourse caudale constituée de deux projections pulpeuses peu cuticularisées à extrémité arrondie, un spi- cule (longueur moyenne $1130 \mu \mathrm{m})$ épais $(10-18 \mu \mathrm{m})$ et à extrémité postérieure arrondie, un cloaque long (moyenne $1423 \mu \mathrm{m}$ ), un canal éjaculateur court (moyenne $167 \mu \mathrm{m}$ ). Une représentation linéaire schématique de l'appareil génital mâle est donnée. La femelle (longueur du corps $11200 \mu \mathrm{m}$ ) présente un appendice vulvaire très variable, présent ou absent selon les spécimens. Les œufs $(64 \mu \mathrm{m} \times 29 \mu \mathrm{m})$ présentent une ornementation réticulée peu visible.

\section{Summary: A new species of capillariin nematode parasitic in soricids (Mammalia, Insectivora) in Nepal.}

Capillaria ohbayashii $\mathrm{n}$. sp. is described from specimens parasitic in the urinary tract of soricid insectivores collected in Nepal, namely Soriculus nigrescens, type host, and Soriculus caudatus, Suncus murinus and Chimarrogalle himalayaca. Males (mean body length $8933 \mu \mathrm{m}$ ) are characterized by the absence of lateral alae, a non-spiny cirrus, a caudal extremity bent ventrally, a caudal bursa made up of two pulpous protrusions slightly cuticularized with round extremities, a thick $(10-18 \mu \mathrm{m})$ spicule(mean length
$1130 \mu \mathrm{m}$ ) with a round posterior extremity, a long cloaca (mean length $1423 \mu \mathrm{m}$ ), a short ejaculatory duct (mean length $167 \mu \mathrm{m}$ ). A linear schematic representation of the male internal reproductive tract is shown. Females (body length $11200 \mu \mathrm{m}$ ) have a very variable vulvar appendage, present or absent depending upon the specimen. Eggs $(64 \mu \mathrm{m} \times 29 \mu \mathrm{m})$ have a reticulate surface ornamentation, feebly visible.

\section{INTRODUCTION}

En 1968, le Professeur Masashi Ohbayashi a collecté au Népal une importante série de parasites d'insectivores soricidés, qui a été communiquée à notre laboratoire. Ce matériel a permis la description de plusieurs espèces nouvelles de Nématodes (Chabaud, 1973; Durette-Desset, 1973). Une espèce nouvelle de Capillariinae est décrite ici.

\section{MATÉRIEL EXAMINÉ}

Hôte-type : Soriculus nigrescens (Gray, 1842); localisation anatomique : vessie urinaire. Les récoltes de M. Ohbayashi comprennent de nombreux lots parasites de cette espèce, parmi lesquels ont été examinés : Lot 306HA, matériel type : dix mâles ( 1 holotype et 9 paratypes) et neuf

Laboratoire de Biologie parasitaire, Protistologie et Helminthologie, URA 114 CNRS, Muséum national d'Histoire naturelle, 61, rue Buffon, F 75231 Paris Cedex 05.

Accepté le: 7 novembre 1991. femelles (1 allotype et 8 paratypes). Localité : Chitare, nordouest de Pokhara, Népal, date 11 mai 1968. Lot 258HA : Trois fragments de femelles et un mâle, détruit pour coupe et examen de la bourse dorsale; même lieu et date que le matériel type. Lot 242 HA : Deux mâles, détruits pour coupe et examen de la bourse dorsale; même lieu et date que le matériel type. Localité : Est de Dunche, Népal, date 31 mai 1968.

Hôte : Suncus murinus (L., 1766), vessie urinaire : Lot 320HA : deux fragments de femelle. Localité : Biratanti, Nord-Ouest de Pokhara, date 13 mai 1968. Lot 311HA : deux fragments de femelle. Localité : Chatrapati, Katmandu, date 23 avril 1968. Lot 323HA : un mâle entier. Localité : Balaju, Katmandu, date 3 juin 1968.

Hôte: Chimarrogale himalayaca (Gray, 1842) (=C. platycephala), vessie urinaire : Lot 264HA : un mâle entier, trois fragments de femelle. Localité : Kuinibisona, Nord de Katmandu, date 7 juin 1968.

Hôte : Soriculus caudatus (Horsfield, 1851), vessie urinaire : Lot 261HA : un fragment de mâle, deux fragments 


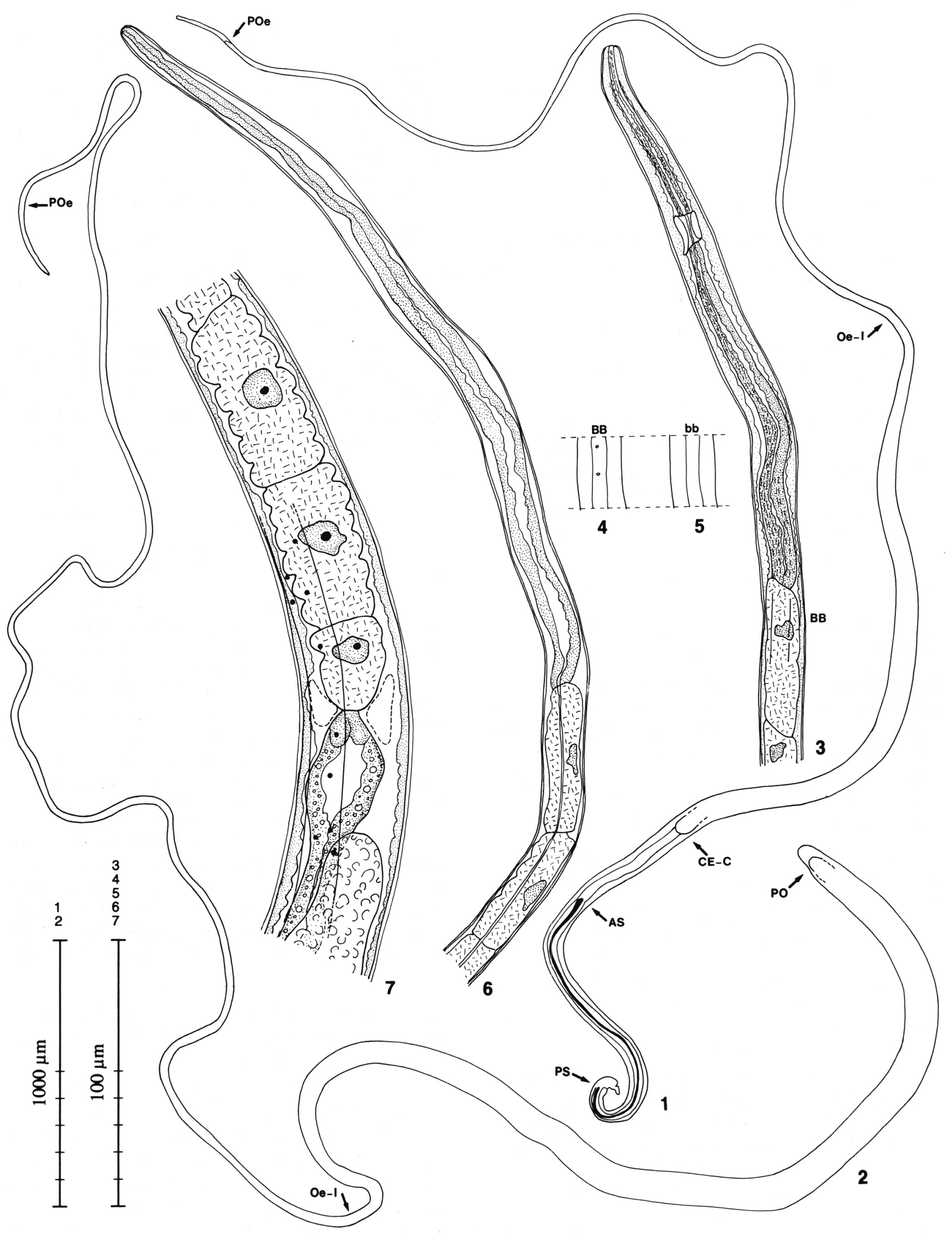

Fig. 1-7. - Capillaria obhayashii n. sp., vues générales, extrémité antérieure, mâle et femelle.

1) Mâle, corps entier ; 2) femelle, corps entier; 3) mâle, extrémité antérieure, préœsophage, deux premiers stichocytes; 4-5) bandes bacillaires au même niveau; 6) femelle, extrémité antérieure, préœsophage, trois premiers stichocytes; anneau nerveux non visible; 7) mâle, jonction œesophage-intestin, bande bacillaire représentée partiellement.

1, 3, 4, 5, 7) holotype; 2, 6) allotype. Tous les dessins sont des vues latérales. 
de femelle. Localité : Chitare, Nord-Ouest de Pokhara, date 11 mai 1968.

\section{DESCRIPTION}

Description basée sur le matériel récolté chez l'hôte-type, Soriculus nigrescens. Toutes les figures sont basées sur le lot 306HA, sauf les figures 14-16, basées sur le lot 242HA.

\section{MÂLES}

(Donné en $\mu \mathrm{m}$ sous la forme : holotype, entre parenthèses : limites de variation chez les paratypes, moyenne)

ASPECTS GÉNÉRAUX (fig. 1, 3) : longueur du corps 8000 (8 700-10 100, moyenne 8933 ). Longueur de la région antérieure (= longueur de l'œsophage) 4000 (3 800-5 600, moyenne 4350 ); plusieurs spécimens sont incomplets dans la région antérieure. Longueur de la région postérieure 4000 (4 100-4 700, moyenne 4 256). Largeur du corps au niveau de la jonction œsophage-intestin 45 (38-50, moyenne 47), au niveau de l'extrémité antérieure du spicule 70 (65-95, moyenne 77), largeur maximum, dans la région postérieure, 90 (95-120, moyenne 103). Longueur du préœsophage 215 (270-290, moyenne 265).

Stichosome (fig. 3,7) : longueur du stichosome 3785 (3 510-5 315, moyenne 4 085). Stichocytes allongés, plus longs que larges, disposés sur une seule rangée. Nombre de stichocytes 46 . Longueur et largeur du dernier stichocyte $90 \times 30(50-70 \times 22-35$, moyenne $64 \times 27)$. Longueur et largeur de l'avant-dernier stichocyte $70 \times 28 \quad(50-70$ $\times 25-35$, moyenne $64 \times 28$ ).

Bandes bacillaires et stries cuticulaires (fig. 3, 4, 5, 7, 9) : deux bandes bacillaires latérales, peu visibles. Pores peu visibles, disposés sur 1-2 rangées irrégulières.

Appareil génital mâle (fig. 7, 8, 10, 11, 12, 13) : extrémité antérieure du testicule au niveau de la jonction œsophage-intestin. Extrémité postérieure du testicule au niveau de la jonction intestin-canal éjaculateur. Longueur totale du testicule (2 635-3 665). Vésicule séminale continuée vers l'arrière par un sphincter (longueur 75) peu musculeux, le canal éjaculateur puis par le cloaque. Longueur du canal éjaculateur 145 (160-180, moyenne 167), largeur 50 (50-70, moyenne 53). Insertion de l'intestin dans le tiers antérieur du canal éjaculateur. Cloaque musculeux. Longueur du cloaque anté-spiculaire 310 (250-300, moyenne 253). Longueur totale du cloaque 1510 (1 310-1 590, moyenne 1 423). Spicule épais, bien sclérifié; extrémité antérieure du spicule peu élargie, avec stries périphériques et pointillés en surface; extrémité postérieure du spicule obtuse, avec stries transversales et pointillés en surface. Longueur du spicule 1150 (1 060-1 200, moyenne
1 130), largeur de l'extrémité antérieure 15 (12-22, moyenne $18)$, de l'extrémité postérieure 10 (8-10, moyenne 10). Cirre inerme, évaginé ou non selon les spécimens, partie évaginée longue de 45 et large de 15 . Spicule évaginé ou non selon les spécimens. Un schéma de l'appareil génital mâle interne, suivant les conventions de Justine (1990), est donné en figure 17.

Extrémité caudale du corps (fig. 10, 12, 13, 14, 15, 16) : ailes latérales absentes. Extrémité caudale enroulée en crosse du côté ventral chez tous les spécimens. Une vue médiane ne peut être obtenue qu'en examinant une extrémité caudale coupée. Bourse caudale formée par deux masses pulpeuses latérales arrondies, chacune portant une projection pulpeuse massive, simple, à extrémité arrondie, orientée dans l'axe longitudinal du corps, souple et faiblement cuticularisée. Largeur de la bourse (48), égale à la largeur du corps dans la partie postérieure.

\section{FEMELLeS}

(Donné en $\mu \mathrm{m}$ sous la forme : allotype, entre parenthèses : limites de variation chez les paratypes, moyenne)

Aspects généraux (fig. 2, 6) : seul l'allotype est entier, et un seul paratype a une région antérieure complète. Longueur du corps 11200 . Longueur de la région antérieure (= longueur de l'œsophage) 7000 (6 200, moyenne 6 600). Longueur de la région postérieure 4200 (3 050-4 800, moyenne 3 992). Largeur du corps au niveau de la jonction œsophage-intestin 42 (50-60, moyenne 51), au niveau de la vulve 50 (50-65, moyenne 57), largeur maximum, dans la région postérieure du corps, 120 (120-145, moyenne 131). Longueur du préœsophage 320 (330, moyenne 325).

Stichosome (fig. 6, 18, 19): longueur du stichosome 6680 (5 870, moyenne 6 275). Stichocytes allongés, plus longs que larges, disposés sur une seule rangée. Longueur et largeur du dernier stichocyte $65 \times 25$ (50-105 $\times 25-30$, moyenne $73 \times 26)$. Longueur et largeur de l'avant-dernier stichocyte $95 \times 25(70-145 \times 25-30$, moyenne $103 \times 27)$.

Bandes bacillaires et stries cuticulaires (fig. 18, 20, 21, $22,23,26,27)$ : deux bandes bacillaires latérales, peu visibles. Pores peu visibles, sur 1-3 rangées irrégulières. Stries cuticulaires transversales peu visibles.

Appareil génital femelle (fig. 18, 19, 21, 24, 25, 28, 29) : vulve avec (fig. 18) ou sans (fig. 19) appendice vulvaire selon les spécimens. Distance extrémité postérieure de l'œsophagevulve 125 (55-200, moyenne 125). Appendice vulvaire formé par une expansion de la cuticule du vagin vrai, fine et souple, longueur de l'appendice vulvaire (100-130), largeur (65-80). OEufs à coque épaisse. Surface de l'œuf avec ornementation réticulée peu visible. Mesure sur 20 œufs chez 7 femelles : Longueur moyenne 64,00 (écart type 2,90), largeur 29,05 (écart type 1,57). 


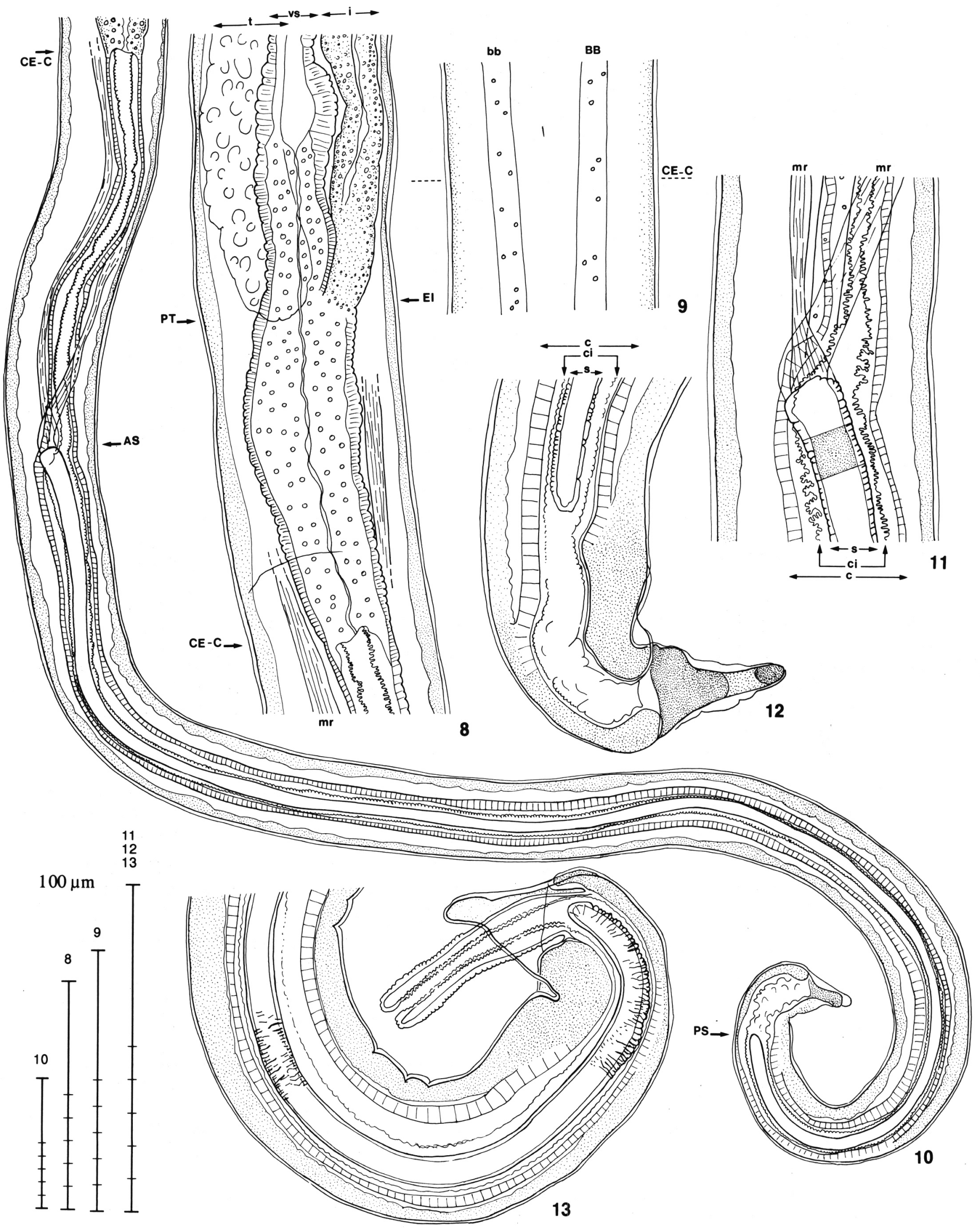



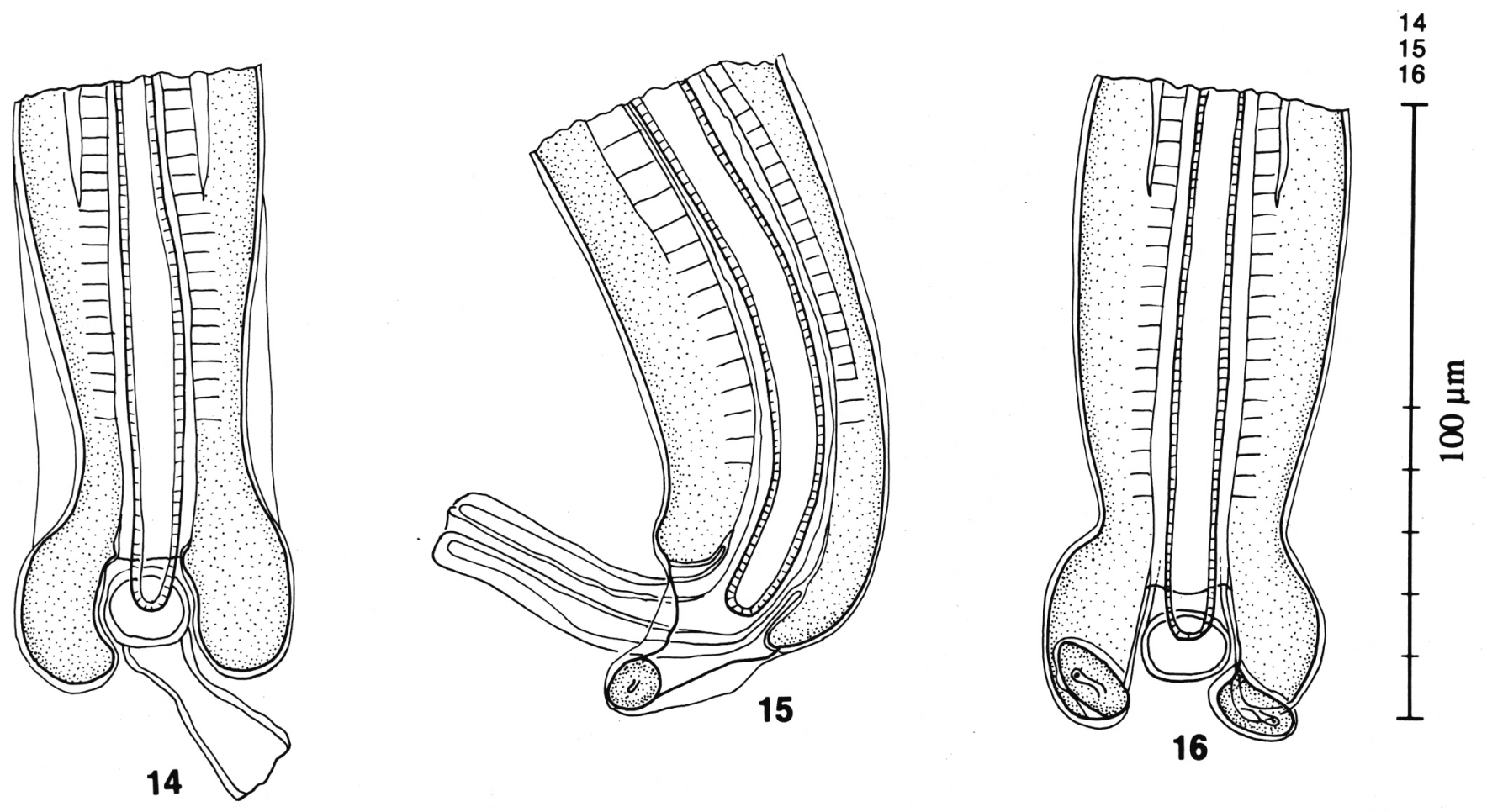

FIG. 14-16. - Capillaria ohbayashii n. sp., mâle : extrémité postérieure du corps coupée pour permettre une vue médiane. Spécimen avec cirre évaginé.

14) vue dorsale, cirre interne non dessiné; 15) vue latérale; 16) vue ventrale, cirre non dessiné, sauf dans sa vue axiale.

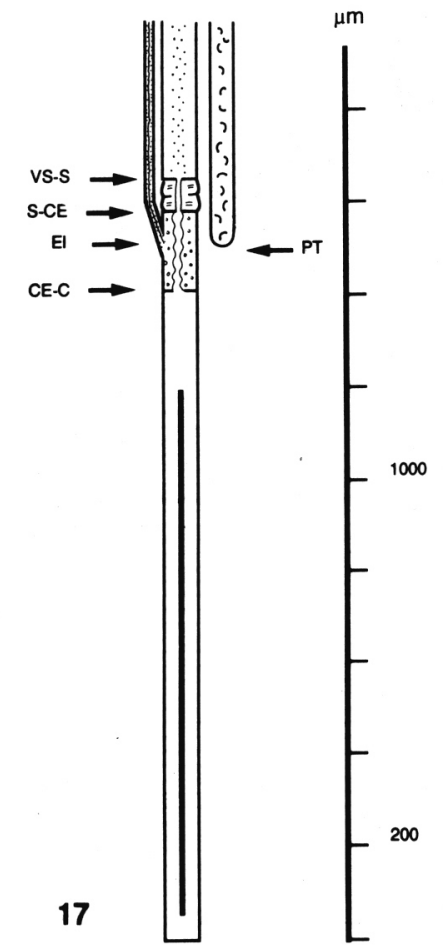

Extrémité caudale du corps (fig. 24, 25) : rectum mal visible; longueur du rectum (65-70, moyenne 68). Distance extrémité postérieure de l'ovaire-extrémité postérieure du corps 45 (35-280, moyenne 76). Importantes variations individuelles dans la distance boucle postérieure de l'oviductequeue : très longue (fig. 24) ou très courte (fig. 25).

\section{MATÉRIEL RÉCOLTÉ CHEZ LES AUTReS HÔTES}

Hôte : Chimarrogale himalayaca. Lot 264HA. Mâle : longueur de la région antérieure 5300 , longueur de la région postérieure 3500 , longueur du spicule 1450 .

Hôte : Suncus murinus. Lot 323HA. Mâle : longueur de la région antérieure 2400 , longueur de la région postérieure 4900 , longueur du spicule 1200 .

Hôte: Soriculus caudatus. Lot 261HA. Fragment de mâle, longueur du spicule 1100 .

Fig. 8-13. - Capillaria ohbayashii n. sp., mâles.

8) Extrémité postérieure du testicule, canal éjaculateur, entrée de l'intestin dans le canal éjaculateur, jonction du canal éjaculateur avec le cloaque; 9) bandes bacillaires au niveau de la jonction canal éjaculateur-cloaque; 10) région postérieure, de l'extrémité antérieure du cloaque à la bourse caudale; 11) extrémité antérieure du spicule, avec deux muscles rétracteurs; surface du spicule et bande bacillaire représentées partiellement; 12) bourse caudale, vue latérale; 13) bourse caudale, vue latérale, chez un spécimen à cirre évaginé; surface du spicule représentée partiellement.

$8,9,10,11)$ holotype; 12, 13) paratypes. Tous les dessins sont des vues latérales. 


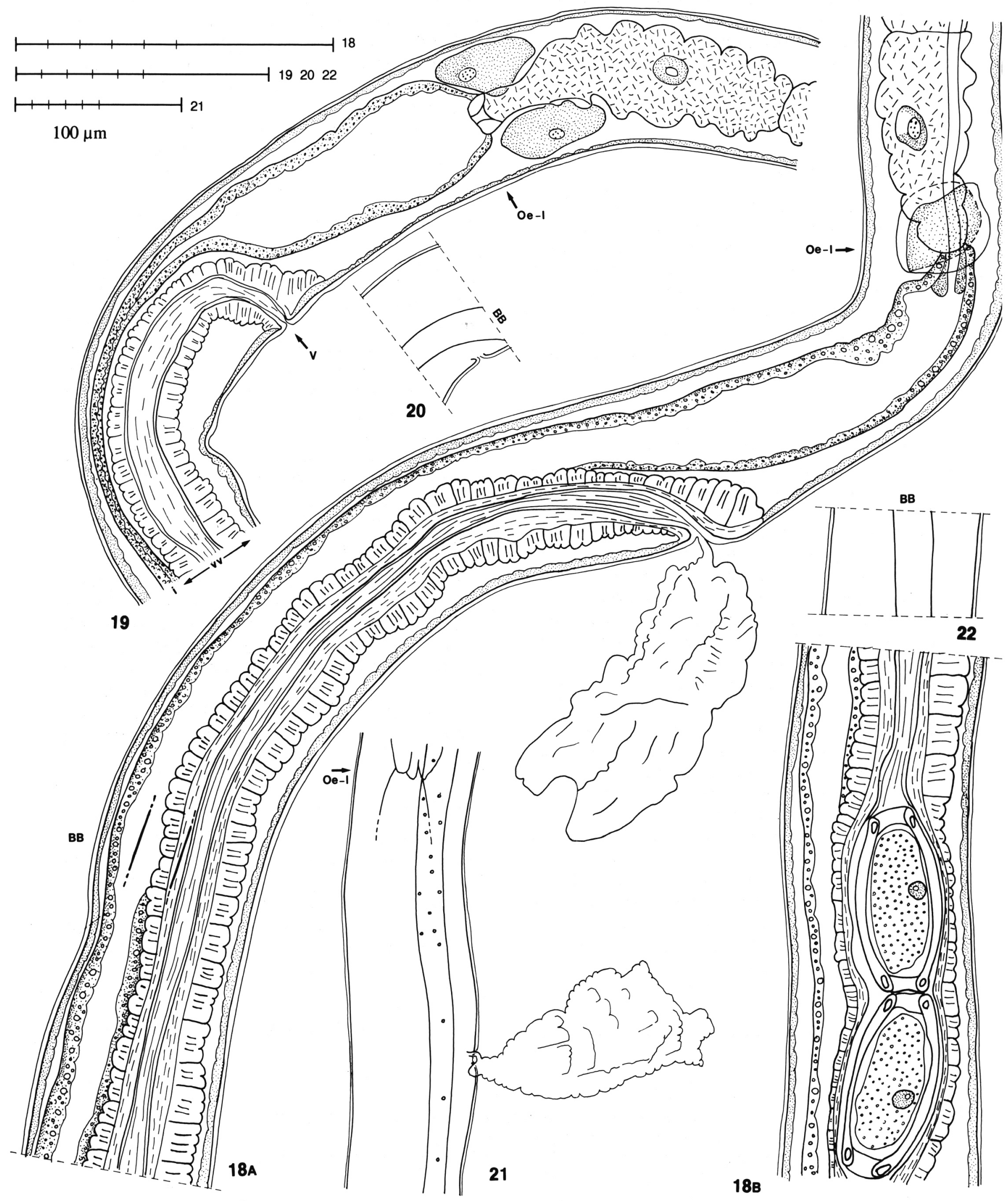


Ce matériel ressemble par sa morphologie aux parasites de Soriculus nigrescens mais n'est pas assez abondant pour permettre une comparaison détaillée. Nous l'assimilons provisoirement à la même espèce.

\section{DISCUSSION}

\section{AtTribution GÉNÉRIQUE}

Jusqu'à vingt-quatre genres sont reconnus chez les Capillariinae par certains auteurs (Moravec, 1982; Baruš et Sergejeva, 1990). D'autres auteurs n'ont pas été convaincus par les arguments proposés pour cette fragmentation et reconnaissent le seul genre Capillaria (par exemple : Anderson et Bain, 1982; Baker, 1987).

La Nématode décrit ici possède les caractères suivants : ailes caudales du mâle absentes, spicule présent, petite bourse membraneuse, cirre non épineux, parasite du système urinaire de mammifères. Dans le système de Moravec (1982), la clé dichotomique des Capillariinae correspondant à ces caractéristiques est : $a$ ) bourse caudale soutenue par deux projections dorsolatérale courtes et rondes, parasites de petits mammifères (rongeurs ou insectivores), genre Liniscus Dujardin, 1845; et $b$ ) bourse caudale soutenue par deux projections étroites, digitées, courbées vers la face ventrale, parasites de carnivores, genre Pearsonema Freitas et Mendonça, 1960. La description du genre dans le texte de Moravec (1982) précise : « extrémité postérieure du mâle portant deux lobes dorsolatéraux très petits ou arrondis; deux projections digitées et très longues présentes, à origine dorsolatérale et recourbée ventralement et vers la ligne médiane, donc supportant la marge de la bourse membraneuse ".

Il y a ici un problème car la description de l'espèce-type du genre Pearsonema, P. pearsoni Freitas et Mendonça, 1960 , ne correspond pas à ces caractéristiques. D'après les figures, $P$. pearsoni a une bourse caudale arrondie, soutenue par deux projections courtes et rondes. Le texte indique : « extrémité postérieure sans ailes latéro-caudales et avec bourse caudale petite, fermée ventralement; dans laquelle se trouvent deux lobes latéro-dorsaux, d'où proviennent deux papilles qui, dirigées latéralement vers la face ventrale, ont une portion apicale atténuée ".

Dans le même article, Freitas et Mendonça proposent d'inclure dans le nouveau genre Pearsonema, d'une part les espèces $C$. linsi, $C$. ransomia et $C$. inflexa, qui ont des bourses caudales à papilles arrondies, et d'autre part, les espèces $C$. feliscati et $C$. linsi, qui ont des bourses à projections latérales très longues et recourbées en hameçon vers la face ventrale. Ce dernier caractère n'est présent ni dans les figures, ni dans le texte de la description originale de Personema pearsoni. Moravec (1982) a classé, en fonction de leur localisation parasitaire, dans le genre Baruscapillaria Moravec, 1982 les espèces parasites du tube digestif à papilles courtes et arrondies, dont $C$. inflexa et $C$. ransomia, et dans le genre Pearsonema les espèces parasites de l'appareil urinaire, dont C. cameroni, C. feliscati et C. linsi. La définition du genre Pearsonema sensu Moravec, 1982 correspond à la morphologie de C. feliscati, C. linsi et $C$. cameroni, mais ne correspond pas à la description originale de l'espèce-type du genre, Pearsonema pearsoni. Elle ne peut être retenue. D'autre part, Moravec (1982) précise que la structure de l'extrémité caudale est mal connue dans certaines des espèces qu'il a regroupées dans le genre Liniscus, et que ce genre demandera une définition plus précise. Je préférerai donc utiliser le système de Anderson et Bain (1982) qui synonymise tous les genres de Capillariinae dans le genre Capillaria, en attendant un système satisfaisant.

\section{INTÉRÊT SYSTÉMATIQUE DE L'APPENDICE VULVAIRE}

Il a été montré que la présence d'un appendice vulvaire peut être très variable à l'intérieur d'une espèce chez les Capillariinae, aussi bien chez des parasites d'oiseaux (Sergeeva, 1979), de poissons (Justine et Radjukovic, 1988), que de mammifères (Justine et De Roguin, 1990). Chez le matériel décrit ici, l'appendice vulvaire est aussi très variable. Le caractère de longueur de l'appendice vulvaire doit donc être utilisé avec prudence dans la systématique des Capillariinae.

\section{AtTRIBUtion SPÉCIFIQUe}

Notre espèce doit être comparée aux Capillariinae parasites de l'appareil urinaire des mammifères et seulement à ceux-là, car ce milieu est suffisamment particulier pour empêcher l'installation de parasites habituellement inféodés à d'autres organes. La liste des 14 espèces regroupée par Moravec (1982) dans les genres Liniscus et Pearsonema a été utilisée, en y ajoutant la nouvelle espèce décrite par

Fig. 18-22. - Capillaria ohbayashii n. sp., femelles : région vulvaire, absence ou présence de l'appendice vulvaire.

18) spécimen avec appendice vulvaire, extrémité postérieure du stichosome, vulve, vagin vrai jusqu'aux deux premiers œufs; dessin en deux parties $18 A$ et $18 B ; 19)$ spécimen sans appendice vulvaire; 20 ) bande bacillaire au niveau de la vulve chez le spécimen dessiné en $19 ; 21$ ) bande bacillaire chez un spécimen à appendice vulvaire; 22) bande bacillaire, $300 \mu \mathrm{m}$ en arrière de la vulve.

$19,20,22)$ allotype; 18, 21) paratypes. Tous les dessins sont des vues latérales. 

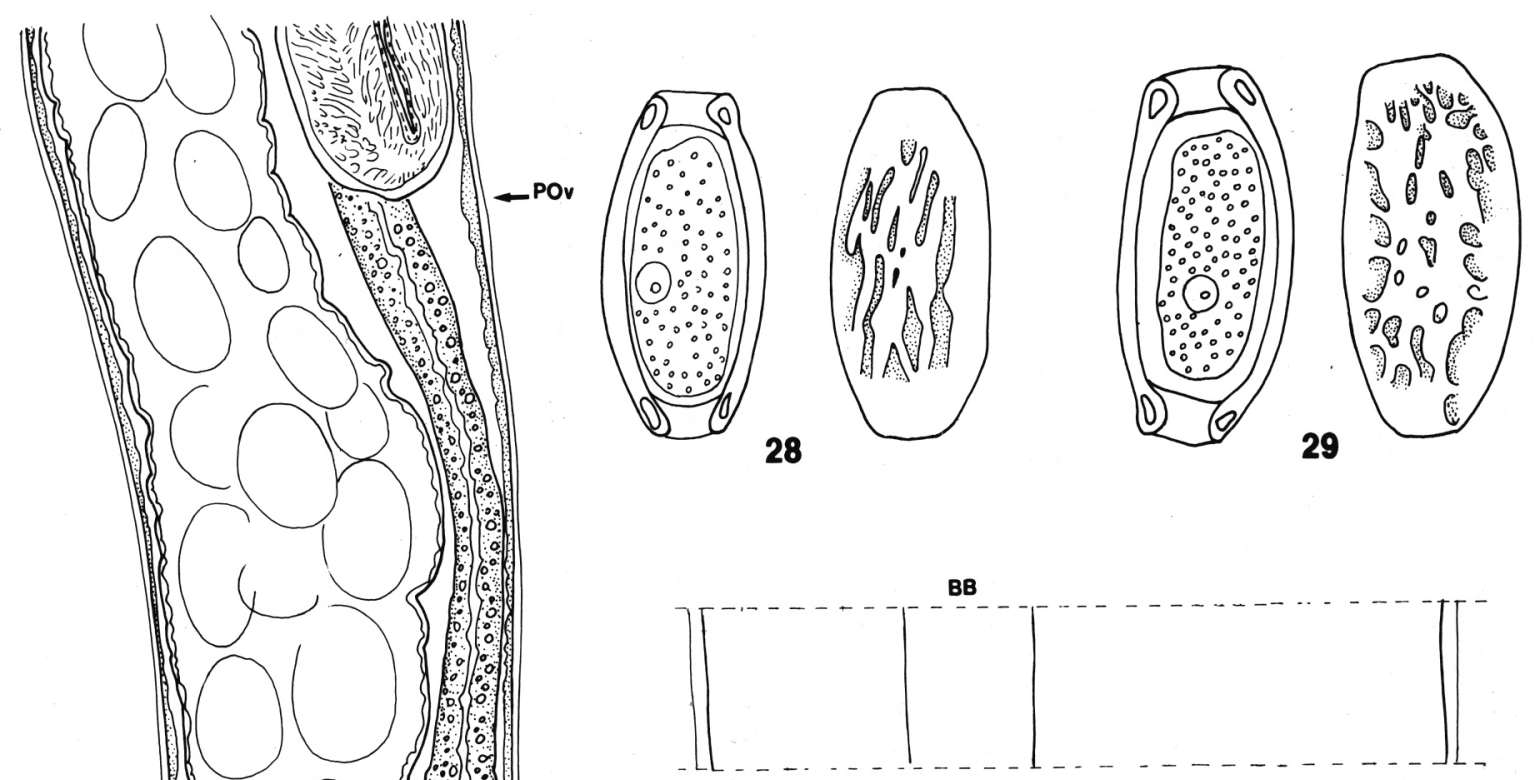

23

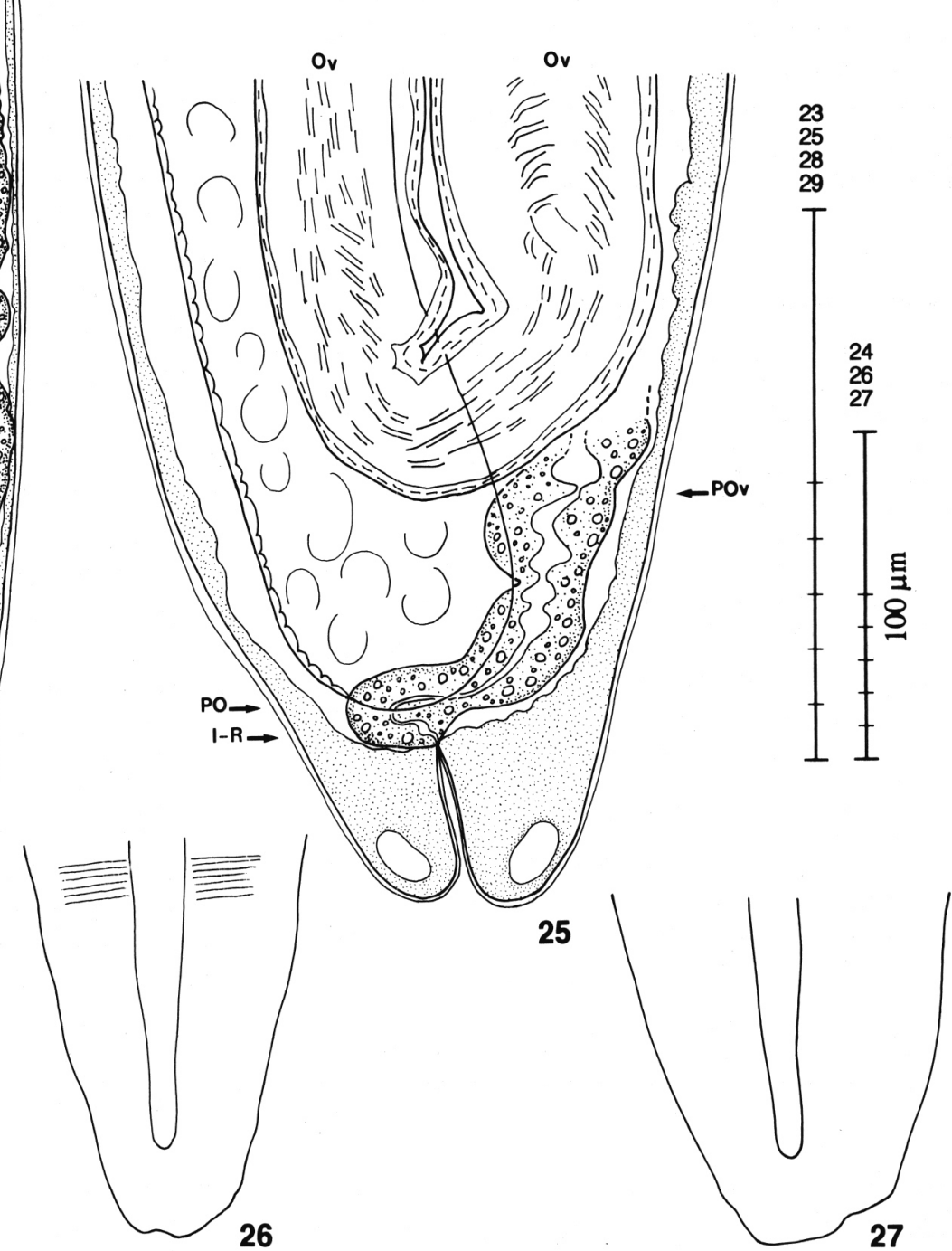

Fig. 23-29. - Capillaria ohbayashii n. sp., femelles : extrémité postérieure, œufs.

23) Bande bacillaire au niveau de la largeur maximale du corps; 24) extrémité postérieure du corps; spécimen avec extrémité de la boucle de l'oviducte éloignée de la queue; 25 ) extrémité postérieure du corps, spécimen avec extrémité postérieure de la boucle de l'oviducte proche de la queue; 26-27) bande bacillaire au niveau de la queue; 28-29) œufs en coupe optique et vue de surface.

23-29) paratypes; 23-27) vues latérales. 
Asakawa, Kamiya et Ohbayashi (1988). Les descriptions des espèces ont été compilées par Freitas et Lent (1936) et Skrjabin, Shikhobalova et Orlov (1957).

Parmi ces espèces, aucune ne possède une bourse caudale mâle similaire à celle de notre matériel. Les espèces qui sont parasites de carnivores ou de rongeurs ont en particulier des bourses caudales très différentes.

Sept espèces sont des parasites d'insectivores (tous soricidés, sauf trois talpidés). Ces espèces ont pour caractère commun des bourses caudales mâles soutenues par des projections courtes et rondes, donc très différentes de celle de notre matériel. Elles diffèrent de plus par d'autres détails. Quatre espèces sont géographiquement éloignées de notre matériel : a) Capillaria capillaris (Linstow, 1882) Stiles et Stanley, 1932, appelée $C$. incrassata par certains auteurs, parasite de Sorex araneus, Crocidura russula et Talpa europea (Talpidé) en Europe, a un spicule long de $810 \mu \mathrm{m}$ (800 à $1150 \mu \mathrm{m}$ selon Wakelin, 1968). b) Capillaria urinicola Soltys, 1952, parasite de Sorex araneus en Pologne a un spicule long de $800 \mu \mathrm{m}$, plus court que notre espèce. c) Capillaria maseri Rausch et Rausch, 1973, parasite de divers Sorex et de Neurotrichus gibbsii (Talpidé) aux USA, a un spicule de taille comparable à notre matériel, mais une ornementation des œufs différente. d) Capillaria reni

Liste des abréviations utilisées dans les figures

AS : niveau de l'extrémité antérieure du spicule/level of the spicule's anterior extremity.

BB : bande bacillaire latérale, vue de surface/lateral bacillary band, surface view.

bb : bande bacillaire latérale, vue par transparence/lateral bacillary band, as seen through the body.

c : cloaque, coupe optique/cloaca, optical section.

CE-C : niveau de la jonction canal éjaculateur-cloaque/level of the ejaculatory duct-cloaca junction.

ci : cirre, coupe optique/cirrus, optical section.

EI : niveau de l'entrée de l'intestin dans le canal éjaculateur/level of the intestine-ejaculatory duct junction.

$\mathrm{i}$ : intestin, coupe optique/intestine, optical section.

I-R : niveau de la jonction intestin-rectum/level of the intestinerectum junction.

$\mathrm{mr}$ : muscle rétracteur du spicule, coupe optique/spicule's retractor muscle, optical section.

Oe-I : niveau de la jonction œsophage-intestin/level of the osophagus-intestine junction.

PO : niveau de l'extrémité postérieure de l'ovaire/level of the ovary's posterior extremity.

POe : niveau de l'extrémité postérieure du préœsophage/level of the preoesophagus' posterior extremity.

POv: niveau de l'extrémité postérieure de la boucle de l'oviducte/level of the oviduct loop's posterior extremity.

PS : niveau de l'extrémité postérieure du spicule/level of the spicule's posterior extremity.

PT : niveau de l'extrémité postérieure du testicule/level of the testis' posterior extremity.

s: spicule, coupe optique/spicule, optical section.

$\mathrm{V}:$ vulve/vulva.

vs : vésicule séminale, coupe optique/seminal vesicle, optical section.

vv : vagin vrai, coupe optique/vagina vera, optical section.
Shaldybin, 1964, parasite de Neomys fodiens en Russie a un spicule long de $720 \mu \mathrm{m}$ et très pointu, à la différence de notre matériel.

Trois de ces espèces ont été décrites en Extrême-Orient, comme notre matériel. a) Capillaria himizu Ohbayashi, Masegi et Kubota, 1972b (= Capillaria sp. Ohbayashi, Masegi et Kubota, 1972a) parasite de Urotrichus talpoides (Talpidé) au Japon, a un spicule long de 384-440 $\mu \mathrm{m}$, donc plus court que notre matériel, et une extrémité caudale du mâle très simplifiée. b) Capillaria hokkaidensis (Asakawa, Kamiya et Ohbayashi, 1988) n. comb (= Liniscus hokkaidensis Asakawa, Kamiya et Ohbayashi, 1988), parasite de Sorex shinto, S. unguiculatus et $S$. minutus au Japon, a un corps et un spicule plus grand que notre matériel, des œufs beaucoup plus petits; l'extrémité caudale du mâle est différente, avec une « projection triangulaire dorsale ». c) Capillaria sunci Chen, 1937, parasite de Suncus coerulus en Chine, Canton et Fujian (Wang, 1982) a des dimensions similaires à celles de notre matériel, mais la description de la bourse caudale mâle est différente de celle de notre matériel.

Au Népal, la seule mention de Capillariinae qui nous soit connue est celle de $C$. dessetae Justine, 1990, qui peut être facilement distinguée de notre matériel par sa morphologie, sa localisation intestinale, et son hôte, un Lagomorphe.

Notre matériel correspond donc à une espèce nouvelle, C. ohbayashii, dédiée à son récolteur, le Professeur Masashi Ohbayashi de l'Université d'Hokkaido au Japon.

\section{CARACTÈRE PRIMITIF DES CAPILLARIINAE PARASITES \\ DE L'APPAREIL URINAIRE DES MAMMIFÈRES}

Anderson et Bain (1982) ont considéré que les Capillariinae les plus primitifs sont les parasites tissulaires. Les espèces parasites de l'appareil urinaire se rattachent à ce groupe, puisque leur localisation implique un passage à travers les tissus, alors que les parasites de l'appareil digestif sont considérés comme plus évolués. Les parasites de l'appareil urinaire présentent des caractéristiques communes : absence d'ailes latérales et bourse caudale mâle petite; ceci peut être considéré comme une morphologie ne permettant la copulation qu'en milieu calme, alors que les espèces soumises au péristaltisme de l'appareil digestif développent des adaptations contraires. Le cirre non épineux est aussi une caractéristique commune de ces espèces de l'appareil urinaire (sauf chez C. longicauda Freitas et Lent, 1935, parasite de l'Opossum) et peut aussi être interprété de la même façon.

D'autre part, le spectre d'hôtes de ces Capillariinae parasites de l'appareil urinaire (Marsupiaux, Insectivores, Carnivores) est composé par des " hôtes anciens » au sens de Chabaud (1981). L'absence d'ailes latérales, une bourse caudale petite, un cirre non épineux doivent donc probablement être considérés comme des caractéristiques primitives chez les Capillariinae. 


\section{RÉFÉRENCES}

Anderson R. C., Bain O. : No. 9. Keys to genera of the superfamilies Rhabditoidea, Dioctophymatoidea, Trichinelloidea and Muspiceoidea. In $\mathrm{CIH}$ Keys to the nematode parasites of Vertebrates (Anderson R. C., Chabaud A. G., Willmott S., eds.), $C A B$, Farnham Royal, 1982, 1-26.

Asakawa M., Kamiya H., Ohbayashi M. : Studies on the parasite fauna of Insectivora. II. Four new capilla:id nematodes from the japanese shrews, genera Sorex and Crocidura. J. Coll. Dairying, 1988, 12, 335-347.

Baker M. R. : Synopsis of the Nematoda parasitic in amphibians and reptiles. Mem. Univ. Newfoundland. Occas. Pap. Biol., $1987,11,1-325$.

Baruš V., Sergejeva T. P. : Capillariids parasitic in birds in the palearctic region (4). Genera Pterothominx and Aonchotheca. Acta Sc. Nat. Brno, 1990, 24, 1-48.

Chen H. T. : New species of Capillaria (Nematoda: Trichuroidea) from the Chinese shrew, Suncus coerulus. Lingnan Sci. J., 1937, $16,149-153$.

Chabaud A. G. : Nouveaux Nématodes Metastrongyloidea parasites d'Insectivores du Népal. Bull. Mus. natn. Hist. nat., Paris, $3^{e}$ sér., 1973, 136, 751-757.

Chabaud A. G. : Spectre d'hôtes et évolution des Nématodes parasites de Vertébrés. In: Deuxième symposium sur la spécificité parasitaire des parasites de Vertébrés, Paris, 13-17 avril 1981. Mém. Mus. natn. Hist. nat, Paris, Sér. A, Zoologie, 1981, 123, 73-76.

Durette-Desset M.-C. : Nouveaux Nématodes Trichostrongyloidea parasites d'Insectivores Soricidés du Népal : description de Suncinema murini $\mathrm{n}$. gen., n. sp., forme relique montrant les liens qui unissent les Molineinae et certains Héligmosomes. Bull. Mus. natn. Hist. nat., Paris, $3^{e}$ sér., 1973, 136, 759-774.

Freitas J. F. Teixeira de, Lent H. : Duas novas especies do genero « Capillaria » Zeder, 1800, parasitas de « Metachirops opossum " (Temm.). Ann. Acad. Brasil. Sci., 1935, 7, 351-353.

Freitas J. F. Teixeira de, Lent H. : Estudo sobre os Capillariinae parasitos de mammiferos (Nematoda: Trichuroidea). Mem. Inst. Oswaldo Cruz, 1936, 31, 85-160.

Freitas J. F. Teixeira de, Mendonça, J. Machado de : Novo nematódeo parasito de Procyon cancrivorus Cuv.: Pearsonema pearsoni gen. nov., sp. nov. (Trichuroidea, Capillariidae). Atas Soc. Biol. Rio de Janeiro, 1960, 4, 63-66.

Justine J.-L., Radujkovic B. M. : Capillaria bainae n. sp. (Nematoda, Capillariinae), parasite du Poisson Parablennius gattoru- gine en mer Adriatique. Bull. Mus. natn. Hist. nat., Paris, 4 éér., 1988, 10, sect. A, 15-24.

Justine J.-L., De Roguin L. : Capillaria murissylvatici (Nematoda, Capillariinae), parasite d'un Rongeur du Baluchistan iranien. Bull. Mus. natn. Hist. Nat., Paris, $4^{e}$ sér., 1990, 12, sect. A, 19-33.

Justine J.-L. : Capillaria dessetae n. sp. (Nematoda, Capillariinae) parasite de Lagomorphe au Népal; intérêt de l'appareil reproducteur mâle interne pour la taxonomie des Capillariinae. System. Parasitol., 1990, 17, 125-132.

Linstow O. : Helminthologische Studien. Arch. Naturgesch., 1882, 48, $1-25$.

Moravec F. : Proposal of a new systematic arrangement of Nematodes of the family Capillariidae. Folia Parasit. (Praha), 1982, 29, 119-132.

Ohbayashi M., Masegi T., Kubota K. : Parasites of the japanese shrew mole, Urotrichus talpoides Temminck. Jap. J. vet. Res., 1972a, 20, 50-56.

Ohbayashi M., Masegi T., Kubota K. : Some nematodes of the japanese shrew mole, Urotrichus talpoides Temminck. Jap. J. vet. Res., 1972b, 20, 111-116.

Rausch R. L., Rausch V. R. : Capillaria maseri sp. n. (Nematoda) from Insectivores (Soricidae and Talpidae) in Oregon. Proc. Helminth. Soc. Wash., 1973, 40, 107-112.

Sergeeva T. P. : The vulval process of Capillaria as a diagnostic sign. Trudy Gel'mintol. Lab., 1979, 29, 129-130 (en russe).

Shaldybin L. S. : Helminth fauna of mammals of the Mordovsk State Reserve. Uchen. Zap. gorkov. gos. pedagog. Inst., 1964, 42 , 52-81 (en russe).

Skrjabin K. I., Shikhobalova N. P., Orlov I. V. : Trichocephalidae and Capillariidae of animals and man and the diseases caused by them. Essentials of Nematodology, vol. VI. (Skrjabin K. I., ed.). Izdatel'stvo Akademii Nauk SSSR, Moscou, 1957. English translation, Israel Program for Scientific Translations, Jerusalem, 1970.

Soltys A. : Pasożyty wewnetrzne ryjówki aksamitnej (Sorex araneus L.) Bialowieskiego Parku Narodowego. Ann. Univ. Mariae Curie Sklodowska (Lublin), 1952, Sect. C, 6, 165-209.

Wakelin D. : Nematodes of the genus Capillaria Zeder, 1800 from the collection of the London School of Hygiene and Tropical Medicine. III. Capillariids from mammalian hosts. J. Helminthol., 1968, 17, 383-394.

Wang P. Q. : Studies on Nematodes of the family Capillariidae from Fujian. Acta Zootaxon. sinica, 1982, 7, 117-126 (en chinois). 